\title{
NON-ITERATIVE ESTIMATION OF THE AC OVERHEAD LINE STATE BY RELINEARIZATION METHOD
}

\author{
Balametov A.B. ${ }^{1 *}$, Khalilov E.D. ${ }^{1}$, Salimova A.K. ${ }^{1}$, Isayeva T.M. ${ }^{2}$ \\ ${ }^{1}$ AzRDPEI, Baku, Azerbaijan \\ ${ }^{2}$ ASOIU, Baku, Azerbaijan
}

\begin{abstract}
Information about the current mode of the electric power system (EPS) is received by the dispatch control centers in the form of telemetry and tele-signals by the SCADA complexes, and from phasor measurement units (PMU) devices. Tele-measurements include information about the mode parameters, and the state of the switching equipment. Since the system of equation of state is nonlinear, the problem of state estimation is traditionally solved using iterative methods. This article presents the results of solving the state estimation problem using a method based on the Kipnis-Shamir re-linearization method, which allows solving it by non-iterative method. The results of the solution are given on the example of a power transmission line with $500 \mathrm{kV}$ voltage.
\end{abstract}

Keywords. measurements, state estimation, PMU, polynomial equations, relinearization

\section{Introduction}

The main trend in development of the modern electric power industry is a control intellectualization. Traditionally, information about the current EPS mode $\mathrm{Y}$ $=\left[\mathrm{P}_{\mathrm{ij}}, \mathrm{Q}_{\mathrm{ij}}, \mathrm{I}_{\mathrm{ij}}, \mathrm{P}_{\mathrm{i}}, \mathrm{Q}_{\mathrm{i}}\right]$ entered the dispatcher control centers in the form of telemetries and tele-signals [1-4].

Since for monitoring the state of the entire power system as a whole, the telemetries from SCADA (Supervisory Control and Data Acquisition) are insufficient and contain errors, for specification of telemetries and calculating unmeasured parameters the state estimation (SE) methods are used.

To monitoring, analysis and operational control of the EPS after SE, the calculation of the steady state (current state) of the electric power systems (EPS) is performed.

In modern conditions the EPS control requires realtime execution of SE of large and complex power systems.

SCADA complexes receive and process distant information once a second, without synchronizing measurements in astronomical time. New measuring equipment - PMU (phasor measurement units) - has been applied with invention of satellite communication systems. Unlike SCADA, PMU measurements are $\mathrm{Y}=$ $\left[\mathrm{U}_{\mathrm{i}}, \mathrm{I}_{\mathrm{ij}}, \delta_{\mathrm{i}}, \varphi_{\mathrm{ij}}\right]$.

Measuring systems for monitoring, control and protection of the power system (WAMS) consisting of PMU devices allow of obtaining a more real state of the power system [5-6].

State estimation of the entire EPS based on the PMU measurements only is currently impossible due to the high cost of the corresponding equipment; therefore, they are usually installed at the most critical facilities.

The mathematical basis of the problem of the SE of EPS is the least square method.

\section{Traditional state estimation}

At classical formulation of the SE problem, the criterion

$$
\varphi(x)=(\bar{y}-y(\hat{x}))^{T} R_{y}^{-1}(\bar{y}-y(\hat{x})) \rightarrow \min ,
$$

is minimized, where $x=(\delta, U)$ is the state vector, consisting of magnitudes $U$ and phase angles $\delta$ of voltages of all nodes of the EPS circuit, except the basic node phase;

$y=f(x)$ - measured mode parameters;

$z=f(x)$ - unmeasured mode parameters;

$R_{y}$ - is a diagonal matrix, the elements of which are the measurement dispersions [1-4].

The state equations are nonlinear, therefore, the SE problem is solved by the iterative method, for example, the weighted least-squares method.

At each iteration, corrections:

$$
\Delta x_{i}=\left[H_{i}^{T} \cdot R_{y}^{-1} \cdot H_{i}\right]^{-1} \cdot H_{i}^{T} R_{y}^{-1}\left[\bar{y}-y\left(x_{i}\right)\right],
$$

and the next approximation $x_{(i+1)}=x_{i}-\Delta x_{i}$ are calculated, where $H$ is measurements Jacobi matrix.

The following initial information can be used as initial approximations of the state vector:

- measurements,

- pseudo-measurements,

- rated values of voltage magnitude and zero values of voltage phases.

Then all unmeasured mode parameters are calculated through the state vector.

To solve the SE problem, the method of test equations (TE) was developed and implemented in the form of program in [2]. The TEs are steady state equations. These equations include measured mode variables and variables calculated through measured ones only.

\footnotetext{
* Corresponding author: balametov.azniie@gmail.com
} 
When PMU and SCADA measurements are used together, the SE problem retains all the disadvantages inherent in traditional state estimate;

- problems in validation due to significant difference in accuracy of PMU and SCADA measurements;

- bad conditionality of the Jacobi matrix and due to this fact, a slowing down of the convergence of the iterative process.

\section{Using of phasor measurements in solving of the EPS SE problem}

Installation of PMU in the EPS nodes allows of using new high-accuracy measurements. At that, the redundancy of measurements increases, which contributes to the detection of glaring errors in telemetries and improves the quality of the state estimation. The main types of measurements received from the PMU are magnitudes and phases of nodal voltages $\left(U_{i}, \delta_{i}\right)$ and currents $\left(I_{i j}, \varphi_{i j}\right)$ in outgoing lines.

Based on the vectors of the nodal voltages and currents of the outgoing lines according to the formulas below, the magnitudes and phases of the nodal voltages in neighboring node scan be calculated:

$$
\begin{aligned}
& \mathrm{U}_{\mathrm{j}}^{\mathrm{cal}}=\sqrt{\mathrm{U}_{\mathrm{i}}^{2}-2 \sqrt{3} \mathrm{U}_{\mathrm{i}} \mathrm{I}_{\mathrm{ij}}\left(\mathrm{r}_{\mathrm{ij}} \cos \left(\delta_{\mathrm{i}}-\varphi_{\mathrm{ij}}\right)-\mathrm{x}_{\mathrm{ij}} \sin \left(\delta_{\mathrm{i}}-\varphi_{\mathrm{ij}}\right)\right)+3 \mathrm{I}_{\mathrm{ij}}^{2}\left(\mathrm{r}_{\mathrm{ij}}^{2}+\mathrm{x}_{\mathrm{ij}}^{2}\right)} \\
& \delta_{j}^{c a l}=\delta_{i}-\operatorname{arctg}\left[\frac{U_{i} \sin \left(\delta_{i}\right)-\sqrt{3} I_{i j}\left(r_{i j} \sin \left(\phi_{i j}\right)+r_{i j} \cos \left(\phi_{i j}\right)\right)}{U_{i} \cos \left(\delta_{i}\right)-\sqrt{3} I_{i j}\left(r_{i j} \cos \left(\phi_{i j}\right)-x_{i j} \sin \left(\phi_{i j}\right)\right)}\right]
\end{aligned}
$$

where, $r_{i j}, x_{i j}$ are the active and reactive resistances of the $\mathrm{i}-\mathrm{j}$ line.

Besides, pseudo-measurements (PM) of power flows can be computed based on the PMU measurements. For example, pseudo-measurements of power overflows at the beginning of a line $P_{i j}^{P M}$ can be calculated as:

$$
\begin{aligned}
& P_{i j}^{P M}=\sqrt{3} I_{i j_{P M U}} U_{i_{P M U}} \cos \phi_{i j_{P M U}}, \\
& Q_{i j}^{P M}=\sqrt{3} I_{i j_{P M U}} U_{i_{P M U}} \sin \phi_{i j_{P M U}},
\end{aligned}
$$

where $I_{i j_{P M U}}$ is current of ij branch; $U_{i_{P M U}}$ is voltage of ith node; $\delta_{\mathrm{ijPMU}}=\left(\delta_{\mathrm{i}}-\delta_{\mathrm{j}}\right)$ is angle between the voltages vectors of $i$ and $j$ nodes.

The initial information for the SE problem is SCADA and PMU measurements, physical and calculated PMU and PM of power flows. The measurement accuracy of the "calculated" PMU is almost equal to the measurement accuracy of the physical PMU. Accuracy of pseudo-measurements of power flows is significantly higher than the accuracy of telemetries in SCADA. This is due to the high accuracy of the PMU measurements. The TEs method by using PMU measurements also allows of checking the quality of SCADA measurements.

For example, when a PMU is installed in separate node, each PMU installed in the node can provide measuring the magnitudes and phase of the voltage in that node and the magnitudes and phases of currents in the outgoing lines. Independent voltage measurements in one node can be used for the validation of these measurements $[1,2]$.

Iterative methods work well for state estimation, but these methods require an initial approximation and can encounter convergence problems if the initial approximation is too far from the actual state of the system.

Large dimension of circuits, complexity and need for the high-speed performance require the development and applying of special algorithms and computational procedures for the SE.

Traditional state estimation methods don't meet the speed requirements. PMU measurements are carried out with a high sampling rate. Therefore, it is possible to estimate the state of individual elements of the EPS (power plants, substations, electrical network zones) in the "rate of process" with very high accuracy.

Linear state estimation of EPS based on PMU measurements is performed in one iteration [6].

In this case, the state vector and the measurement vector are, respectively, equal to

$$
x=\left[\begin{array}{l}
\dot{U}_{i}=U_{i}^{\prime}+j \cdot U_{i}^{\prime \prime} \\
\dot{U}_{j}=U_{j}^{\prime}+j \cdot U_{j}^{\prime \prime}
\end{array}\right], \quad \bar{y}=\left[\begin{array}{l}
\dot{U}_{i}=U_{i}^{\prime}+j \cdot U_{i}^{\prime \prime} \\
\dot{U}_{j}=U_{j}^{\prime}+j \cdot U_{j}^{\prime \prime} \\
\dot{I}_{i j}=I_{i j}^{\prime}+j \cdot I_{i j}^{\prime \prime} \\
\dot{I}_{j i}=I_{j i}^{\prime}+j \cdot I_{j i}^{\prime \prime}
\end{array}\right],
$$

The measurement vector is related to the EPS state vector as $\bar{y}=H \cdot x$, where

is the Jacobi matrix.

$$
H=\left[\begin{array}{cc}
1 & 0 \\
0 & 1 \\
\dot{Y}_{i j}+\dot{Y}_{i 0} & -\dot{Y}_{i j} \\
-\dot{Y}_{i j} & \dot{Y}_{i j}+\dot{Y}_{j 0}
\end{array}\right]
$$

The linear state vector is calculated as:

$$
x=\left[B^{T} R^{-1} B\right]^{-1} B^{T} R^{-1} Z .
$$

\section{State estimation based on re- linearization method}

The SE considered in [7] is based on the Kipnis-Shamir relinearization method [7]. In this method measurement equations, which are the voltage value at the node of the power line and the equations for the driving power of the node are formulated using rectangular coordinates of the bus voltages. At such formulation nonlinear measurement equations become quadratic voltage polynomials [8]. Then the method uses two transformations of the original system to the highdimensional equations system with the quadratic variables to solve using non-iterative method. At accurate measurements this method gives the same results as the weighted least squares method. 
The initial data required for the method is the system topology, information about the mode parameters and measurements from the system.

If the transmission line parameters are expressed using the $\pi$-model and the measurements are voltage magnitudes and linear flows, then the measurement equations have the form [8-9]:

$$
\begin{gathered}
U_{i}^{2}=U_{i R}^{2}+U_{i I}^{2} ; \quad U_{j}^{2}=U_{j R}^{2}+U_{j I}^{2} ; \\
\left.P_{i, j}=g_{i, j}\left(U_{i R}^{2}+U_{i I}^{2}-U_{i R} U_{j R}\right)-U_{i I} U_{j I}\right) \\
+b_{i, j}\left(U_{i I} U_{j R}-U_{i R} U_{j I}\right) ; \\
Q_{i, j}=b_{i, j}\left(U_{i R}^{2}+U_{i I}^{2}-U_{i R} U_{j R}-U_{i I} U_{j I}\right) \\
+g_{i, j}\left(U_{i R} U_{j I}-U_{i I} U_{j R}\right) \\
+b s\left(U_{i R}^{2}+U_{i I}^{2}\right) \\
g_{i j}=\frac{R_{i j}}{Z_{i j}^{2}}, b_{i j}=
\end{gathered}
$$

where $\mathrm{i}$ is the sending node; node $\mathrm{j}$ is the active power receiving node, $\mathrm{R}_{\mathrm{ij}}, \mathrm{X}_{\mathrm{ij}}$, and $\mathrm{b}_{\mathrm{s}}$ are the active resistance of line, reactance and conductivity to earth, respectively.

The nodal power equations are constructed by summing all the linear flow equations that come out from the node plus the power flowing into any external active conductance $\mathrm{g}$ or reactive conductance $\mathrm{b}$ connected to the node (for example, shunt capacitor or reactor).

$$
P_{i}=\sum P_{i j}+g_{i} U_{i}^{2} ; \quad Q_{i}=\sum Q_{i j}+b_{i} U_{i}^{2} .
$$

where $\mathrm{j}$ is the set of nodes connected to node $\mathrm{i}$.

Since these equations are linear with respect to the quadratic voltage terms $\left(U_{i R}^{2} ; U_{i I}^{2} ; U_{i R} U_{j R}\right.$, etc. $)$, they can be represented in matrix form

$$
A_{\xi} \xi=C,
$$

where $\mathrm{C}, \xi, \mathrm{A}_{\xi}$-are the vector of measured values, the vector of quadratic voltage variables, and the matrix of coefficients for $\xi$, respectively. The vector $\xi$ consists of the quadratic variables of the real and imaginary parts of the voltages denoted by $\mathrm{x}_{\mathrm{i}} \mathrm{x}_{\mathrm{j}}$, where the indexes $\mathrm{i}$ and $\mathrm{j}$ are not associated with the numbers of the nodes.

\section{First transformation of variables}

Transformation of variables is performed, and system (4) is rearranged in the following form

$$
[A B]\left[\begin{array}{l}
Y \\
Z
\end{array}\right]=C
$$

where A contains linearly independent columns $A_{\xi}$, and $\mathrm{B}$ contains the remaining columns $\mathrm{A}_{\xi}, \mathrm{Y}$ is vector of elements $\xi$ corresponding to $\mathrm{A}$, and $\mathrm{Z}$ is vector of elements corresponding to $\mathrm{B}$.
Let's denote the quadratic variables $\mathrm{x}_{\mathrm{i}} \mathrm{x}_{\mathrm{j}}$ in $\mathrm{Y}$ as $\mathrm{y}_{1}$; $\mathrm{y}_{2} ;,,, ; \mathrm{y}_{\mathrm{Ny}}$, and by $\mathrm{N}_{\mathrm{y}}$ we denote the total number of variables $Y$. Quadratic variables $x_{i} x_{j}$ in $Z$ we denote as $\mathrm{z}_{1} ; \mathrm{z}_{2} ;,,, ; \mathrm{z}_{\mathrm{Nz}}$ in the order and through $\mathrm{N}_{\mathrm{z}}$ the total number of $Z$-variables.

In addition, all quadratic variables containing the imaginary component of the balancing node and the corresponding columns of the matrix are excluded from the system, in the balancing node since a zero imaginary component is specified.

The $Y$ variables in the rearranged system can now be expressed in terms of $\mathrm{Z}$ variables and measurement values $\mathrm{C}$ :

$$
\begin{gathered}
Y=d+D \cdot Z \\
d=\left(A^{T} A\right)^{-1} A^{T} C \\
D=-\left(A^{T} A\right)^{-1} A^{T} B
\end{gathered}
$$

The set of equation takes the following form:

$$
\left[\begin{array}{c}
y_{1} \\
\cdot \\
\vdots \\
y_{N_{y}} \\
z_{1} \\
\vdots \\
\vdots \\
z_{N_{Z}}
\end{array}\right]=E \cdot\left[\begin{array}{c}
1 \\
z_{1} \\
\vdots \\
z_{N_{z}}
\end{array}\right]
$$

where the new matrix $E$ is formed by combining $d$ and $D$ with the subsequent addition of the zero column and the unit matrix I of $\mathrm{N}_{\mathrm{z}}$ size.

$$
E=\left[\begin{array}{ll}
d & D \\
0 & \\
\vdots & I \\
0 &
\end{array}\right]
$$

In this matrix $\mathrm{d}$ is the only part of $\mathrm{E}$ that depends on the measured values of $\mathrm{C}$. It should be noted that $\mathrm{A}$ and $\mathrm{B}$ are determined by the topology and parameters of the system, and therefore D and, accordingly, the unit matrix drops below it. This means that as long as the topological system remains unchanged, it is only necessary to recalculate $d$ when a new series of measurements is received and the remaining parts of $\mathrm{E}$ can be taken from the previous calculations.

\section{Second transformation of variables}

At this stage combinations of paired products of quadratic variables are formed according to certain rules. Correct paired products meet the condition:

$$
\mathrm{s}_{\mathrm{ij}} \mathrm{S}_{\mathrm{pq}}=\left(\mathrm{x}_{\mathrm{i}} \mathrm{x}_{\mathrm{j}}\right)\left(\mathrm{x}_{\mathrm{p}} \mathrm{x}_{\mathrm{q}}\right)=\left(\mathrm{x}_{\mathrm{i}} \mathrm{x}_{\mathrm{p}}\right)\left(\mathrm{x}_{\mathrm{j}} \mathrm{x}_{\mathrm{q}}\right)=\mathrm{s}_{\mathrm{ip}} \mathrm{S}_{\mathrm{jq}}
$$

These pair product ratios are used to impose additional constraints on the unknowns so that a correct 
solution can be obtained. For the paired products to be valid, the $\mathrm{s}_{\mathrm{ij}}$ and $\mathrm{s}_{\mathrm{pq}}$ must exist among the set of quadratic variables $\mathrm{Y}$ and $\mathrm{Z}$, and the $\mathrm{s}_{\mathrm{ij}}$ and $\mathrm{s}_{\mathrm{pq}}$ can not be the same pair as $s_{\text {ip }}$ and $s_{j q}$. For each correct paired product, one equation can be generated in the form: $\mathrm{s}_{\mathrm{ij}} \mathrm{S}_{\mathrm{pq}}-\mathrm{S}_{\mathrm{ip}} \mathrm{S}_{0}$. More details on this can be found in [8].

\section{Simulation}

The simulation was carried out for $350 \mathrm{~km}$ long $500 \mathrm{kV}$ power transmission line (PTL) (see fig.1). In [10], simulation of the mode of such line was carried out by using the equations of the line with distributed parameters.

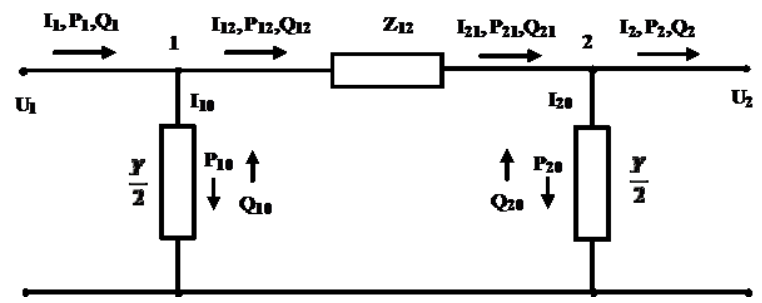

Figure: Two-node $\pi$-scheme of the PTL

For this example, the voltage values at both nodes are known. The active (P) and reactive (Q) power flux measurements from node 1 to node 2 , are obtained from four measurement equations. Node 1 is set as balancing one, and therefore its imaginary component becomes 0 , as a result of which three unknown voltage components must be determined from the four measurement equations.

OHL parameters in relative units $\mathrm{R}=0.0046 ; \mathrm{X}=$ $0.04186 ; \mathrm{g}=0.014 ; \mathrm{b}=3.2$.

The measurements are: $\mathrm{U} 1=520.06 \mathrm{kV} ; \mathrm{U} 2=490$ $\mathrm{kV}$; $\mathrm{P} 12=935.18 \mathrm{MW}$; $\mathrm{Q} 12=80.07$ MVAr.

The results of calculation of the steady state of the PTL are presented in the table.

Table. Results of steady-state of the PTL

\begin{tabular}{|c|c|c|c|c|c|c|}
\hline Bus & Voltage & Ang & \multicolumn{2}{|c|}{ Generation } & \multicolumn{2}{c|}{ Load } \\
\hline & $\begin{array}{c}\text { Mag } \\
(\mathrm{pu})\end{array}$ & $(\mathrm{deg})$ & P, MW & $\begin{array}{c}\text { Q, } \\
\text { MVAr }\end{array}$ & $\begin{array}{c}\text { P, } \\
\text { MW }\end{array}$ & $\begin{array}{c}\text { Q, } \\
\text { MVAr }\end{array}$ \\
\hline 1 & 1.042 & 0 & 935.18 & 80.07 & 0 & 0 \\
\hline 2 & 0.973 & -22.64 & 0 & 0 & 900 & 50 \\
\hline
\end{tabular}

\begin{tabular}{|c|c|c|c|c|c|c|}
\hline \multirow[t]{2}{*}{ Branch } & \multirow[t]{2}{*}{$\begin{array}{c}\text { From } \\
\text { Bus }\end{array}$} & \multirow[t]{2}{*}{$\begin{array}{c}\text { To } \\
\text { Bus }\end{array}$} & \multicolumn{2}{|c|}{$\begin{array}{l}\text { From Bus } \\
\text { Injection1 }\end{array}$} & \multicolumn{2}{|c|}{ To Bus Injection 2} \\
\hline & & & $\begin{array}{l}\text { P, } \\
\text { MW }\end{array}$ & $\begin{array}{c}\text { Q, } \\
\text { MVAr }\end{array}$ & $\begin{array}{l}\text { P, } \\
\text { MW }\end{array}$ & $\begin{array}{c}\text { Q, } \\
\text { MVAr }\end{array}$ \\
\hline 1 & 1 & 2 & 935.18 & 80.07 & -900 & -50 \\
\hline
\end{tabular}

Total power losses are: $\quad 35.184 \mathrm{MW} \quad 362.76 \mathrm{MVAr}$

The bus admittance matrix is

YBUS $=\left(\begin{array}{cc}2.30242-22.03152 \mathrm{i} & -2.29542+23.66652 \mathrm{i} \\ -2.29542+23.66652 \mathrm{i} & 2.30242-22.03152 \mathrm{i}\end{array}\right)$
Forming of measurement matrixes and coefficients C, A, B, d, D are:

$$
\mathrm{CM}:=\left[\begin{array}{c}
(\mathrm{U} 1 \mathrm{M})^{2} \\
(\mathrm{U} 2 \mathrm{M})^{2} \\
\mathrm{P} 12 \\
\mathrm{Q} 12
\end{array}\right]=\left(\begin{array}{c}
1.08576 \\
0.94673 \\
9.3518 \\
-0.8007
\end{array}\right) \mathrm{B}:=\left(\begin{array}{l}
0 \\
1 \\
0 \\
0
\end{array}\right)
$$

$$
\begin{aligned}
\mathrm{A} & :=\left(\begin{array}{cccc}
1 & 0 & 0 & 0 \\
0 & 1 & 0 & 0 \\
\text { YBUSR11 } & 0 & - \text { YBUSR12 } & - \text { YBUSI12 } \\
- \text { YBUSI11 } & 0 & - \text { YBUSI21 } & - \text { YBUSR22 }
\end{array}\right)= \\
& =\left(\begin{array}{cccc}
1 & 0 & 0 & 0 \\
0 & 1 & 0 & 0 \\
2.2954 & 0 & -2.2954 & -23.6665 \\
22.0303 & 0-23.6665 & 2.2954
\end{array}\right)
\end{aligned}
$$

$$
\begin{array}{r}
\mathrm{D}:=-\left(\mathrm{A}^{\mathrm{T}} \cdot \mathrm{A}\right)^{-1} \cdot \mathrm{A}^{\mathrm{T}} \cdot \mathrm{B}=\left(\begin{array}{c}
0 \\
-1 \\
0 \\
0
\end{array}\right) \\
\mathrm{d}:=\left(\mathrm{A}^{\mathrm{T}} \cdot \mathrm{A}\right)^{-1} \cdot \mathrm{A}^{\mathrm{T}} \cdot \mathrm{C}=\left(\begin{array}{c}
1.08576 \\
0.94673 \\
1.02607 \\
-0.19032
\end{array}\right)
\end{array}
$$$$
\left(\begin{array}{cccc}
1 & 0 & 0 & 0 \\
0 & 1 & 0 & 0 \\
2.31542 & 0 & -2.31542 & -23.66652 \\
22.03027 & 0 & -23.66652 & 2.31542
\end{array}\right) \cdot \text { ynew }=\mathrm{C}-\mathrm{Bz}
$$

Then the solution of the system of equations is represented as

$$
\text { Ynew }=\left(\begin{array}{c}
1.08576 \\
0.81314 \\
1.02607 \\
-0.19032
\end{array}\right)
$$

Then the systems of the following equations are solved to find $t$

$$
\operatorname{At} 1 \cdot\left(\begin{array}{l}
1 \\
\mathrm{z}_{1} \\
\mathrm{z}_{1} \\
\left(\mathrm{z}_{1}\right)^{2}
\end{array}\right)=\left(\begin{array}{l}
0 \\
0
\end{array}\right) \text {, }
$$




$$
\text { At1 }:=\text { Find(At1) }=\left(\begin{array}{cccc}
0.15749 & 0 & -1.17888 & 0 \\
-0.15749 & 0 & 1.17888 & 0
\end{array}\right)
$$

The elements of this matrix are used to compose the matrixes $\mathrm{k}$ and $\mathrm{A}_{\mathrm{t}}$

$$
\mathrm{k}=\left(\begin{array}{c}
0.15749 \\
-0.15749
\end{array}\right) \quad \text { At }=\left(\begin{array}{c}
-1.17888 \\
1.17888
\end{array}\right)
$$

to solve the system $A t \cdot t=k$ from which one can find $\mathrm{t}$ and, $\mathrm{z}$ accordingly,

$$
\begin{aligned}
& \mathrm{z}_{1}=0.13359 \\
& \mathrm{t}_{1}=0.13359
\end{aligned}
$$

Passing to the original designations, we get:

$$
\begin{gathered}
\mathrm{xSE}_{1}:=\sqrt{\mathrm{ynew}_{1}}=1.042 \\
\mathrm{xSE}_{2}:=\sqrt{\text { ynew }_{2}}=0.90174 \\
\mathrm{xSE}_{3}:=\delta 1=0 \\
\mathrm{xSE}_{4}:=-\sqrt{\mathrm{z}_{1}}=-0.3655
\end{gathered}
$$

\section{Conclusion}

The advantage of the SE based on the relinearization method is that it does not have the disadvantages of the convergence problem.

Compared to traditional weighted least squares method, the non-iterative method requires more measurements for observability. The traditional method can solve cases with 3 or more measurements. This is because the non-iterative method tries to compute the solution, while the least squares method repeats in the direction of the solution.

The results of applying the method on $500 \mathrm{kV}$ PTL is presented.

\section{References}

1. Schweppe F.C., Wildes J. Power system static state estimation. Part 1: exact model. // IEEE Trans. On Power Systems, №1, 1970. -pp.120-125.

2. Gamm A.Z. Statistical methods for assessing the state of electric power systems // M.: Nauka, 1976. pp.17-24.

3. Evaluation of the state in power engineering / A.Z Gamm, L.N. Gerasimov, I.I. Golub et al. / Edited by Yu.N. Rudenko, Moscow: Nauka, 1983, 320 p.

4. Gamm A.Z., Glazunova A.M., GrishinYu.A., Kolosok I.N., Korkina E.S. Development of algorithms for assessing the state of the electric power system // Electricity. - 2009. - No. 6. - pp.2-9.
5. A.G
Phadke.
Synchronized
Phasor Measurements. A Historical Overview.// IEEE/PES
Transmission and Distribution Conference, 2002, vol. 1, pp.476-479

6. A.G. Phadke and J.S. Thorp, Synchronized Phasor Measurements and Their Application, Springer Science + Business Media, 2008.

7. Kipnis A, Shamir A. Cryptanalysis of the HFE public key cryptosystem. In: Proceedings of Crypto '99. Santa Barbara (CA); 1999. pp.19-30.

8. Fardanesh B. Method and systems for power systems analysis: a non-iterative state solver/estimator for power systems operation and control. U.S. Patent 20 050160 128; January 31, 2012.

9. Jiang XT, et al. Power system state estimation using a non-iterative direct state calculation method. Presented at CURENT annual site visit and industry conference, Knoxville, TN; 2013.

10. Balametov A., Khalilov E., Isayeva T.M. On the increasing of accuracy of power transmission lines modes mathematical modeling. Proceedings of the 6th International Conference on Control and Optimization with industrial Applications, Baku, Azerbaijan, pp.98101. 